\title{
EL PARQUE CULTURAL DE VALLTORTA-GASULLA (CASTELLÓN)
}

\author{
THE CULTURAL PARK OF VALLTORTA-GASULLA (CASTELLÓN)
}

RAFAEL MARTÍNEZ VALLE (*)

\section{RESUMEN}

Se expone el proceso que ha llevado a la creación del Parque Cultural de Valltorta-Gasulla, uno de los espacios más singulares de la Comunidad Valenciana por la importancia de sus manifestaciones rupestres prehistóricas. Con la creación del Parque Cultural, figura legal incluida en la Ley de Patrimonio Cultural Valenciano, se pretende desarrollar un modelo de gestión integral del Patrimonio Cultural.

\begin{abstract}
The paper explains how the Cultural Park of ValltortaGasulla was created. The Park has become one of the most significant places of the Valencian area, due to its important prehistoric rock art. With the creation of the Cultural Park, itself a legal item included in the Valencian Heritage Law, the intention was to develop an integral managerial model for the Cultural Heritage.
\end{abstract}

Palabras clave: Arte Rupestre Levantino. Prehistoria. ComunidadValenciana. Conservación. Investigación. Difusión.

Key words: Levantine Rock Art. Prehistory. Valencian Region. Conservation. Research. Diffusion.

\section{INTRODUCCIÓN}

Las comarcas de Els Ports y El Maestrat, en el norte de Castellón, constituyen uno de los territorios de la Comunidad Valenciana más ricos en

(*) Museu de la Valltorta. Generalitat Valenciana. Partida Pla del'Olm s/n. 12179 Tirig. Castellón.

El artículo fue remitido en su versión final el 6-XI-2000. manifestaciones rupestres prehistóricas. Desde el año 1916, fecha que marca el descubrimiento de las pinturas de Morella La Vella (Hernández Pacheco, 1918) hasta la actualidad, el número de conjuntos inventariados no ha cesado de incrementarse.

En este amplio territorio destacan por la densidad de conjuntos y por su singularidad, dos cuencas hidrográficas: el Barranc de La Valltorta y la Rambla Carbonera en cuya cabecera se encuentra el Barranc de Gasulla (Fig. 1).Actualmente existen en ambos espacios 33 conjuntos, pertenecientes en su mayoría al denominadoArte Levantino, manifestación incluida desde diciembre del año 1998 en la lista del Patrimonio Mundial de la UNESCO.

En este territorio la Dirección General de Promoción Cultural y Patrimonio Artístico de la Generalitat Valenciana ha iniciado el primer Parque Cultural con arte rupestre de nuestra Comunidad.

\section{LOS CONJUNTOS RUPESTRES DE LA VALLTORTA Y EL BARRANC DE GASULLA}

La historia de La Valltorta comienza el año 1917 con el descubrimiento de la Cova dels Cavalls y otros importantes conjuntos por Albert Roda. El hallazgo tuvo hondas repercusiones en el ámbito científico y en el contexto social regional.

A comienzos del año 1917 se desplazaron hasta el barranco tres comisiones de estudio; una delegación de la Comisión de Investigaciones $\mathrm{Pa}$ leontológicas y Prehistóricas dirigida por Hugo Obermaier que llevó a cabo el estudio y publicación de los principales conjuntos localizados en el sector oeste de la Valltorta (Obermaier y Wernert, 1919), otra del Institut d’Estudis Catalans bajo la tutela de P. Bosch Gimpera que además de comenzar el estudio de los conjuntos rupestres del sector 
este inició un proyecto de estudio de los yacimientos arqueológicos del entorno de los abrigos pintados (Duran i Sempere, 1915-20) y Joan Cabré, quien como delegado del Marqués de Cerralbo llevaría a cabo la documentación de los principales conjuntos de La Valltorta (Cabré, 1923) y el estudio pormenorizado de les Coves de Ribasals o del Civil (Cabré, 1925).

A la repercusión científica del hallazgo de este importante conjunto de pinturas rupestres se sumaron otros efectos: una inusitada curiosidad entre la población de áreas limítrofes que acudían hasta la Valltorta para conocer las pinturas rupestres y el
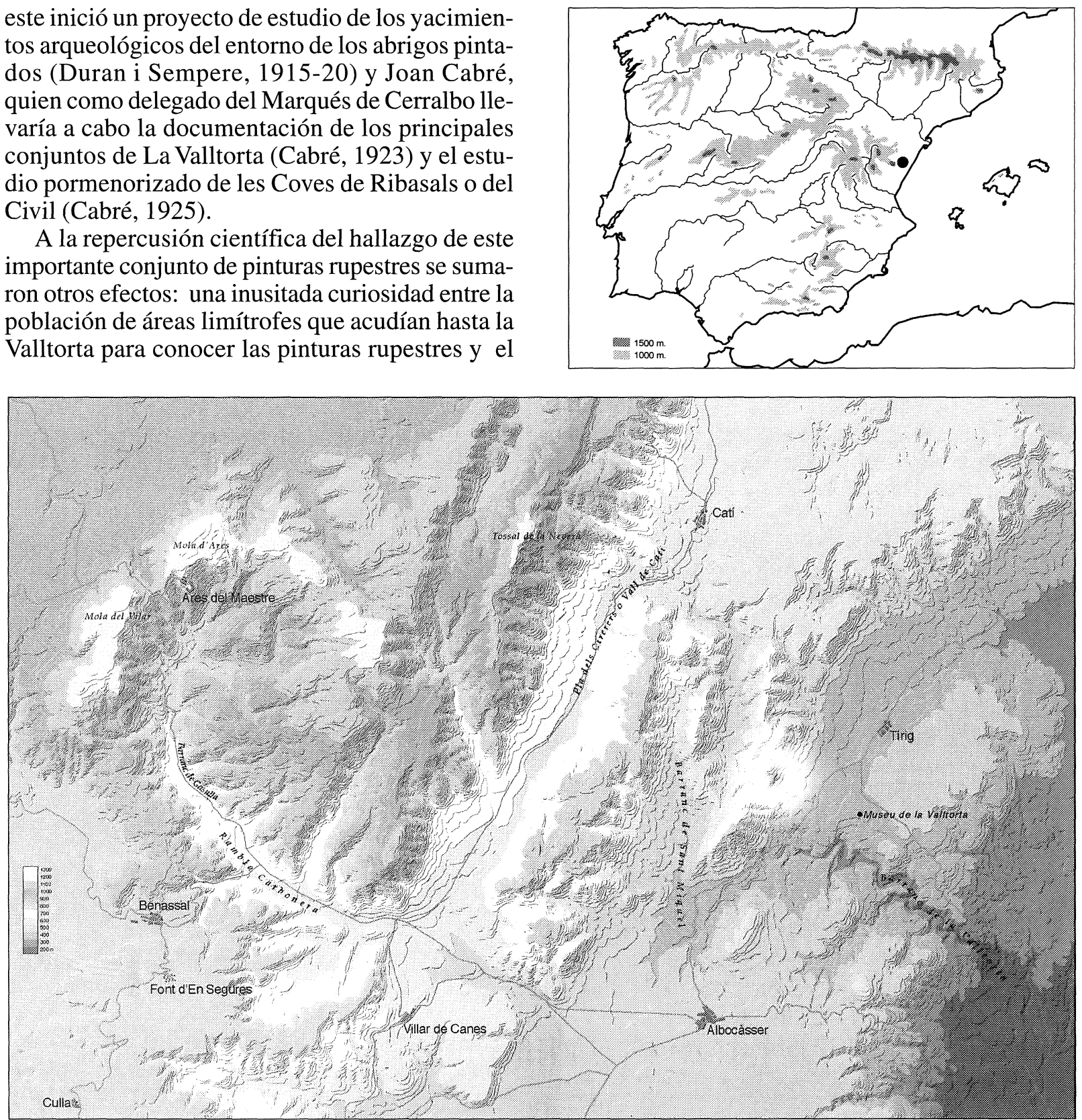

Fig. 1. Situación del Parque Cultural de Valltorta-Gasulla (Castellón) en la Península Ibérica y mapa del Parque Cultural con localización de los principales conjuntos de arte rupestre.

malestar de una parte de la población local ante semejante movimiento de gentes. El resultado de esta afluencia incontrolada fue la destrucción parcial de algunos conjuntos (Cabré, 1923). El propio Obermaier se lamenta en la monografía de la Valltorta del deterioro sufrido por algunos abrigos (Obermaier y Wernert, 1919: 77) en estos términos: los contemporáneos y la posteridad sabrán juzgar tales extremos de vandalismo por el que fueron criminalmente destruidos tesoros únicos, que no solo para España sino para el mundo entero, representan monumentos históricos insubstituibles.

El proceso de deterioro consecuencia del vandalismo y las visitas incontroladas se prolongó hasta el año 1942, fecha en que se contrató un vigilante para custodiar el conjunto (Viñas, 1982). No obs- 
tante no se abandonaron determinadas prácticas lesivas como el mojado reiterado de los paneles pintados para avivar los colores, o los graffitis recordatorios de las visitas.

En estos años la Valltorta es un referente obligado en los estudios de arte prehistórico. Este reconocimiento contrasta con la escasez de estudios llevados a cabo en las décadas siguientes, tanto de sus conjuntos rupestres a los que se dedicarán estudios puntuales (Beltrán, 1965; Ripoll, 1970), como también de sus yacimientos arqueológicos (Maluquer, 1938; Almagro, 1944). En los años setenta se produce una reactivación en los estudios de La Valltorta. El Servicio de Investigación Arqueológica y Prehistórica de la Diputación Provincial de Castellón, creado el año 1975, llevará a cabo excavaciones en el Cingle de L'Ermitá (Gusi, 1975a) y en la Cova del Mas d'Abad (Gusi, 1975b; Gusi y Olaria, 1975); M. ${ }^{\mathrm{a} J}$. de Val (1977) realizará su Tesis de Licenciatura sobre las industrias líticas dels Planells y Viñas (1970, 1978, 1979-80, 1981) iniciará sus estudios sobre sus conjuntos rupestre.

La historia del núcleo de Gasulla discurre por similares derroteros. El año 1934 tenía lugar el descubrimiento de la Cova Remigia (Ares, Castellón) $\mathrm{y}$ otros conjuntos igualmente importantes en su entorno (Porcar, 1934, 1935). Cova Remigia fue estudiada por Porcar, Obermaier y Breuil (1935) y publicada de forma modélica.

Pese a esta transcendencia el inicio de la Guerra del 36 favoreció un menor conocimiento de estos lugares. Los trabajos desarrollados por Porcar (1945, 1946, 1947, 1949a, b, c) en los años siguientes dan cuenta de la importancia de este núcleo en el contexto del Arte Levantino peninsular. El estudio del Cingle de la Mola Remigia, que iniciara Breuil el año 1934 y que no concluyó a causa de su precario estado de salud, será finalizado por Ripoll (1963) años después.

La compra del conjunto de Cova Remigia y el Cingle por la Diputación de Castellón y el establecimiento de un servicio de guardería evitaron los niveles de deterioro alcanzados por los cercanos conjuntos de La Valltorta.

En la década de los setenta, al igual que ocurriera en La Valltorta, se reanuda el interés por los yacimientos arqueológicos próximos a las pinturas. La información recopilada por Porcar (1934) servirá de base a trabajos más amplios. González Prats (1979) lleva a cabo el inventario arqueológico de la comarca delAlt Maestrat, donde incluye informa- ción de los yacimientos del Barranc de Gasulla y comienzan las investigaciones sobre el controvertido yacimiento de La Cova Fosca (Aparicio Pérez y San Valero, 1977; Mesado Oliver, 1981; Olaria, 1988).

\section{EL PARQUE CULTURAL DE LA VALLTORTA}

En la década de los años 80 el deterioro de ambos conjuntos, y muy especialmente el del Barranc de la Valltorta, había llegado a unos extremos alarmantes como consecuencia de una escasa vigilancia y un flujo de visitantes cada vez más numeroso y desordenado.

El año 1983, recién asumidas las competencias autonómicas por la Generalitat Valenciana, se puso en marcha un proyecto de gestión integral dirigido a atajar sus problemas de conservación. El proyecto de creación del Parque Cultural de la Valltorta fue diseñado desde los Servicios Territoriales de la Consellería de Cultura en Castellón. Con él se pretendía un doble objetivo: proteger el Barranc de la Valltorta, uno de los lugares más ricos en manifestaciones rupestres de toda la Comunidad Autónoma e incentivar el desarrollo local mediante el turismo cultural.

En la primera mitad del año 1984 se esbozó el proyecto de actuaciones redactado por el técnico de los Servicios Territoriales de Castellón J.L. Constante Lluch. El proyecto estuvo marcado por la interdisciplinaridad y por el intento de hacer converger a diversas instituciones (ICONA, IRYDA (1), Consellerias de Agricultura y de Cultura, Ayuntamientos). En el equipo de trabajo se integraban Ramón Viñas como especialista en arte rupestre, Arturo Zaragoza como arquitecto y el propio Juan L. Constante como geógrafo.

La propuesta se articulaba en 4 grandes apartados: Protección Jurídica, Protección Física, Equipamientos y Difusión Cultural.

A) Protección Jurídica. Se pretendía dotar al Barranc de la Valltorta de protección legal, salvaguardando las manifestaciones rupestres y evitar las agresiones paisajísticas que pudieran incidir en el estado adquirido por el conjunto en su propia evolución histórica. Con la legislación del momento se

(1) Instituto para la Conservación de la Naturaleza e Instituto para la Reforma y el Desarrollo Agrario (Ministerio de Agricultura). 
recurrió a la Ley de Patrimonio de 1933 y a la Ley de Espacios Naturales Protegidos de 1975. De acuerdo con ambas la Valltorta sería un Conjunto Histórico Artístico y Espacio Natural Protegido, en alguna de sus modalidades. Conocedores del Proyecto de Ley de Patrimonio, en ese momento debatiéndose en las Cortes se esperaba poder declarar $\mathrm{La}$ Valltorta como Sitio Histórico.

Esta protección se concretaba en la delimitación de tres ámbitos (zonas 1, 2 y 3). La zona 1 incluía el propio Barranc de la Valltorta y sus principales afluentes, donde se localizan los abrigos con arte rupestre. En este ámbito debía mantenerse inalterado el paisaje. La zona 2 incluía la ladera oriental de Montegordo y las laderas a ambos lados del barranco. Aquí se preconizaba la continuidad de los cultivos de secano y el mantenimiento de las construcciones existentes. Finalmente la zona 3 sería una aureola en torno a las anteriores a fin de amortiguar los impactos sobre las áreas protegidas.

B) Protección física. Se planteó la necesaria protección de los abrigos pintados mediante la construcción de cerramientos integrados en el paisaje y la creación de un servicio de vigilantes.

C) Equipamientos. Se proyectó la construcción de un núcleo cultural-recreativo, con instalaciones y servicios de variada funcionalidad, entre ellas un museo, un camping, restaurantes, áreas de esparcimiento. Estos servicios de complementaban con inversiones en una presunta restauración del paisaje, que incluía la creación de vallados para fauna silvestre, la repoblación forestal de parcelas abandonadas con pinos (Pinus halepensis) y la apertura de viales de tráfico rodado y senderos para aproximarse a los conjuntos de arte rupestre. Todo debía gestionarse a través de un patronato que nunca llegó a constituirse.

Las primeras respuestas frente al proyecto fueron variadas pero en general negativas. La población local vio en la propuesta de la administración un obstáculo a determinadas iniciativas agrícolas y ganaderas en curso como las transformaciones del secano tradicional en regadío o la construcción de granjas porcinas. Desde sectores más minoritarios se planteó que el turismo de masas era incompatible con la conservación de LaValltorta y sus pinturas rupestres.

El resultado fue el abandono del proyecto de creación del Parque. No obstante continuó el proyecto de construcción de un museo y parte de los equipamientos así como la protección física de los principales conjuntos.

\section{EL PROYECTO DEL PARQUE CULTURAL DE VALLTORTA-GASULLA}

\section{1. ¿Qué es un Parque Cultural?}

La propuesta formulada el año 1984 para La Valltorta aspiraba a gestionar los sitios con arte rupestre y sus respectivos entornos sin excluir ningún elemento del paisaje, con independencia de su monumentalidad o cronología. Se pretendía una aproximación al paisaje, como objeto de aprendizaje y disfrute, incidiendo en las relaciones del hombre y el medio desde la prehistoria hasta la actualidad. Fue una propuesta innovadora, deudora en alguna medida de los primeros ecomuseos franceses (Riviere, 1980).

Con la legislación al uso difícilmente se podía proteger un extenso territorio desde una perspectiva exclusivamente cultural, de ahí que se propusiera la incardinación de dos figuras legales el Sitio Histórico y el Paisaje Protegido.

Con la promulgación de la Ley 16/85 de 25 de Junio del Patrimonio Histórico Español se avanza hacia una protección más efectiva del arte rupestre. En el artículo 40.2 se establece que las cuevas, abrigos y lugares que contengan manifestaciones de arte rupestre quedan declaradas Bienes de Interés Cultural (BIC), consideración que conlleva la necesaria delimitación de entornos de protección.

En años posteriores el Departamento de Cultura de la Dirección General de Aragón organizó reuniones de trabajo en Albarracín (1986), Barbastro (1987) y Zaragoza (1989) donde se volvió a plantear la necesidad de diseñar un estrategia de protección para los territorios con arte rupestre.

La reunión de Zaragoza (AAVV, 1989) sirvió para poner en común diversas experiencias nacionales y extranjeras y para debatir sobre problemas legales en el manejo de estos espacios. En este encuentro Morell (1990) define dos aspectos esenciales de los Parques Culturales: el del espacio o territorio valorizado por la existencia del arte rupestre y en su función como contenedor y en ese sentido museo de estas manifestaciones.

Esta noción de entorno valorizado por el BIC difiere del concepto manejado en numerosas actuaciones sobre inmuebles, en las que el entorno es una unidad de protección y su delimitación se ha de fundamentar en base a las exigencias de protección de los mismos (Castillo Ruiz, 1997).

En su segunda condición, el Parque Cultural como Museo, coincide con uno de los rasgos de los

T. P., 57, n. $^{\circ} 2,2000$ 
Parques Arqueológicos tal y como son definidos por Querol (1989), no obstante se presenta como una figura más integradora. En el Parque Cultural el patrimonio arqueológico puede ser un elemento esencial pero no siempre será el determinante.

Los Parques Culturales participan de ambas características: espacios valorados por los BIC y consideración de museos y aspiran a incorporar de forma activa a la población que habita el territorio. Esta perspectiva, presente también en los ecomuseos, se ha visto reforzada en los últimos años con las demandas sociales a favor del desarrollo sostenible, puestas de manifiesto en la Conferencia de Río (1992) y en diversa normativa europea entre las que cabe destacar la emanada de la IV Conferencia de los Ministros responsables del Patrimonio Cultural de los países miembros del Consejo de Europa (Helsinki, 1996), donde se insiste en el papel del Patrimonio Cultural como factor de desarrollo sostenible.

En este contexto deben inscribirse las iniciativas autonómicas aragonesa y valenciana de redacción de sendos cuerpos legales que regulan los Parques Culturales.

La Ley 12/1997 de 3 de Diciembre de Parques Culturales de Aragón ha sido elaborada desde las competencias de Patrimonio Cultural si bien constituye un instrumento de ordenación del territorio rural. La ley define el Parque como un territorio que contiene elementos relevantes de Patrimonio Cultural, integrados en un marco físico de valor paisajístico y/o ecológico singular.

En el caso valenciano, el año 1998 se promulgó la Ley 4/1998 de 11 de Junio, de la Generalitat Valenciana del Patrimonio Cultural Valenciano en la que se incluye la figura del Parque Cultural en la categoría de Bien de Interés Cultural de carácter inmueble. Este queda definido como el espacio que contiene elementos significativos del patrimonio cultural integrados en un medio fisico relevante por sus valores paisajísticos y ecológicos.

\subsection{Los límites de la propuesta}

El proyecto de Parque Cultural que iniciamos el año 1998 presenta como principal novedad respecto al proyecto de 1984 la inclusión junto al Barranc de la Valltorta de los conjuntos rupestres del núcleo de Gasulla. Esta decisión se basa en la transcendencia patrimonial de los conjuntos localizados en este área, especialmente Cova Remigia y el Cingle y en

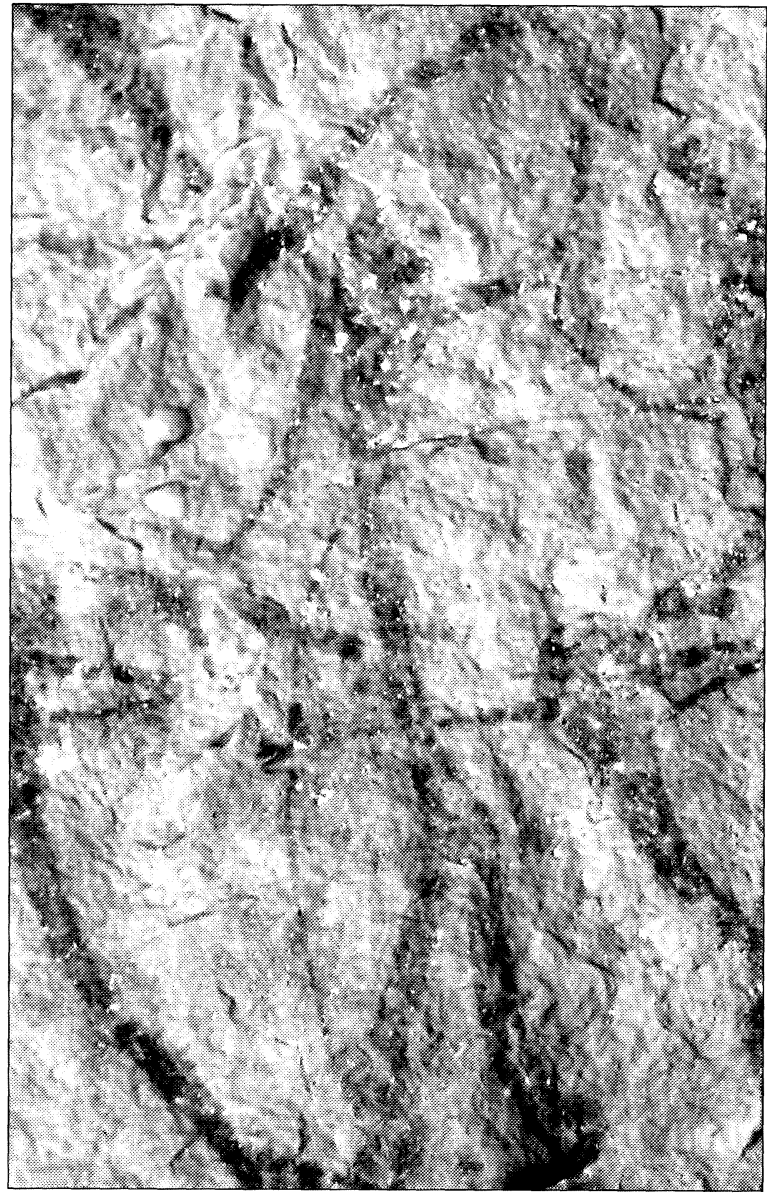

Lám. I. Detalle de la escena principal del abrigo III de les Coves de Ribasals o del Civil, Barranc de la Valltorta (Tírig).

la continuidad geográfica documentada en la distribución de los conjuntos pintados.

El Parque de Valltorta-Gasulla afecta a una extensión aproximada de 22000 ha y a ocho términos municipales (Fig. 1). Las variables conjugadas en la delimitación de este espacio han sido:

A) Las manifestaciones rupestres. La existencia de una distribución continua de abrigos con arte rupestre en ambas cuencas con una densidad claramente superior a la observada en áreas próximas. Esta elevada densidad de pinturas rupestres, pertinente desde criterios arqueológicos, posibilita llevar a cabo un proyecto integral de gestión que incluya su estudio como expresión más directa de las comunidades prehistóricas, su protección y una adecuada difusión (Lám. I).

B) Los yacimientos arqueológicos. Un elevado número de yacimientos arqueológicos, en muchos 


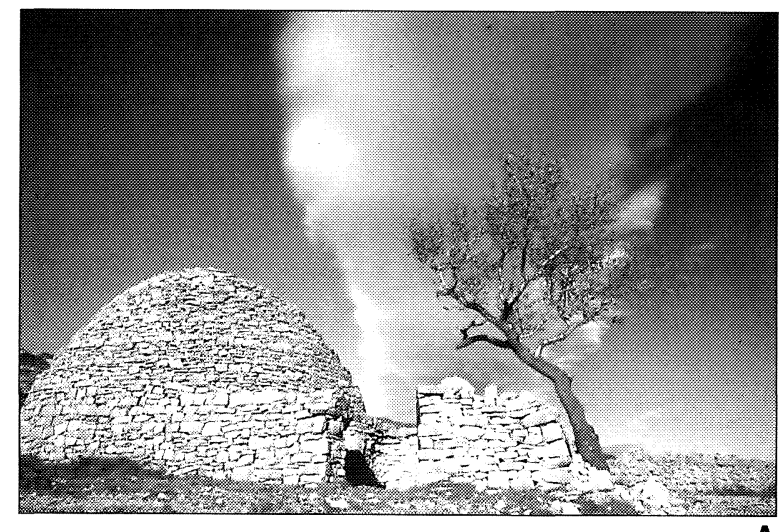

A

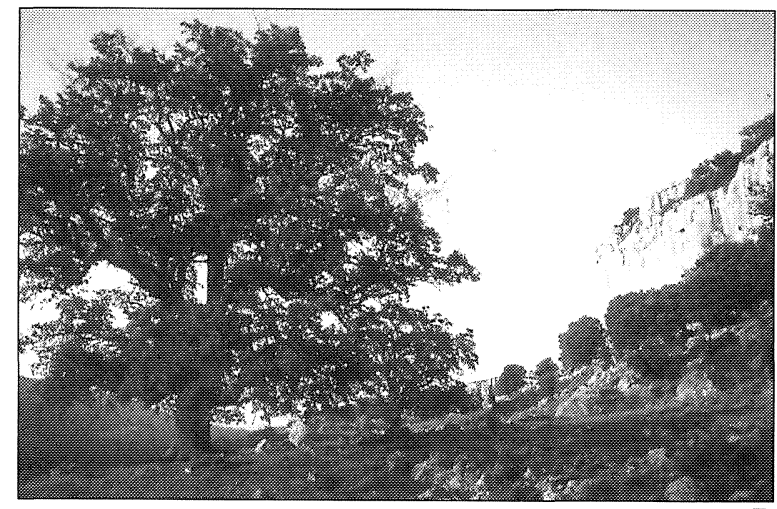

B

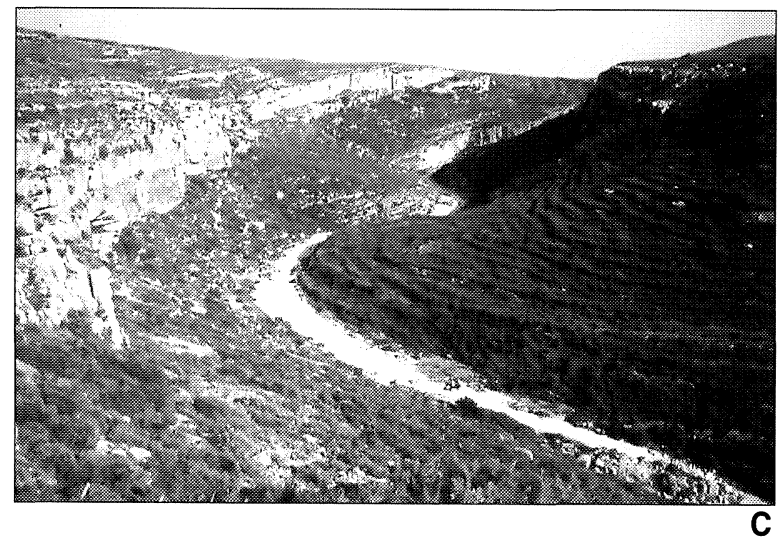

Lám. IIA) Construcción en piedra seca, Caseta de volta del Barranc de Gatella (Catí), B) Roble centenario del Barranc dels Horts (Ares); C) Vista general del Barranc de la Valltorta con los abrigos de La Saltadora al fondo.

casos claramente relacionables con los conjuntos pintados.

C) El medio rural. La localización de pinturas rupestres y yacimientos arqueológicos en un entorno cultural tradicional basado en el aprovechamiento agropecuario del medio, con una población dispersa en núcleos familiares, las masías, con las que se asocia toda una serie de construcciones en piedra seca ligadas a los aprovechamientos citados ( $\mathrm{ca}$ setas de volta, azagadors, pous, cenies, neveres) (Lám. II A).

D) La integración de estos elementos en ecosistemas soporte de una variada vegetación y fauna.

\subsection{Medio físico}

El Parque se extiende por una zona de media montaña perteneciente al Sistema Ibérico. Su sector occidental se incluye en el dominio tabular maestracense, caracterizado por relieves amesetados de alturas superiores a los $1200 \mathrm{~m}$ surcados por profundos y escarpados barrancos vertientes a la Rambla Carbonera. Es un paisaje calizo en el que encontramos todas las formaciones típicas del modelado cárstico. La altitud y unas precipitaciones superiores a los $600 \mathrm{~mm}$ anuales favorecen el desarrollo de carrascales continentales (Hedero helicis-Quercetum rotundifoliae). En las zonas más elevadas, el pastoreo y las duras condiciones climáticas han favorecido el establecimiento de pastizales y formaciones almohadilladas de altura (ErodioErinacetum) y carrascales con sabina negral (Junipero thuriferae-Quercetum rotundifoliae). En los barrancos más abrigados existen bosquetes relictos de roble valenciano(Violo willkommii-Quercetum rotundifoliae) algunos de cierta extensión como el conservado en el Barranc del Horts, con ejemplares centenarios (Lám. II B).

La fauna existente en este sector es muy variada. Entre los mamíferos destacamos la presencia de la cabra montés(Capra pyrenaica hispanica), el jabalí (Sus scropha) y abundantes carnívoros. La comunidad de aves es igualmente variada además de las especies comunes del bosque mediterráneo encontramos numerosas especies protegidas como el Buitre leonado(Gyps fulvus), el águila real (Aquila chrysaetos) y el búho real (Bubo bubo).

El paisaje del Parque cambia en la medida que descendemos hacia la costa. El relieve influido por las alineaciones Costero Catalanas toma una orientación NO/SE. En este sector encontramos amplios corredores paralelos a la costa delimitados por sierras de escasa altura por los que discurren los afluentes que vierten al Barranc de laValltorta (Lám. II C). Es un paisaje profundamente antropizado. En el fondo de los valles se asientan las principales poblaciones (Albocàsser, Cati, Tirig, Les Coves de Vinromà) y a su alrededor se extienden los cultivos de 
secano. En algunos barrancos como el de LaValltorta se conservan retazos de carrascales litorales con palmito (Rubio longifoliae-Quercetum rotundifoliae) y encuentran refugio especies muy escasas como el águila perdicera (Hieraetus fasciatus).

\subsection{Medio humano}

El área propuesta ha sufrido, al igual que otros territorios rurales de la Comunidad Valenciana, un despoblamiento acelerado en los últimos decenios, más acentuado en los periodos comprendidos entre los años 1910-1930 y entre 1960 y 1975 y con mayor influencia en la población concentrada que en la dispersa (Baila Pallares, 1990).

Respecto a la estructura de edades se ha producido en envejecimiento progresivo importante. El año 1994 la zona contaba con 5000 habitantes de los que unos 1300 eran mayores de 64 años.

Un rasgo definitorio del territorio analizado es la importancia de la población dispersa. El año 1970 era superior al 25\% (valores máximos en la ComunidadValenciana).Actualmente ha disminuido pero todavía constituye un porcentaje elevado. De esta circunstancia depende el que las construcciones rurales se hayan mantenido en buen estado, y que algunas de ellas se hayan restaurado y transformadas en alojamientos para el turismo rural.

La conservación de esta población dispersa es uno de los objetivos del Parque, que debe materializarse con apoyos institucionales que compatibilicen la mejora de sus condiciones de vida con la conservación del paisaje.

La agricultura es la actividad principal de esta población, destacando municipios como Tírig, Vilar de Canes y Ares donde la población activa dedicada a la agricultura ronda el 70\%. En el Parque existen 4400 ha de cultivos, la mayor parte de secano (olivos, almendros, cereales, forrajes, avellanos y algarrobos en las tierras bajas). Estas explotaciones agrarias tradicionales, en regresión en comarcas limítrofes, constituyen uno de los principales valores del Parque Cultural.

Respecto a la actividad industrial solamente destacan dos poblaciones Benassal, con una planta envasadora de aguas y Catí con una fabrica de piensos y otra de muebles. Con la excepción de estas actividades, el resto de la población activa industrial se dedica a las granjas de cría intensiva de ganado porcino.

La tradición ganadera ha experimentado un brus-

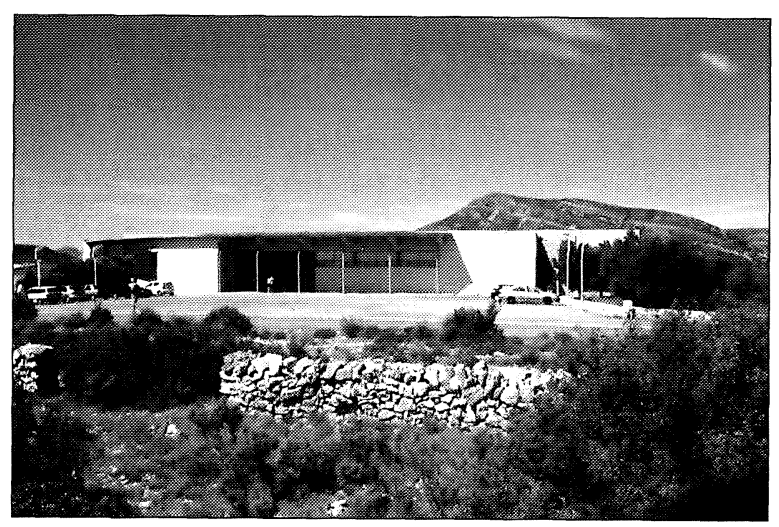

Lám. III. Museu de la Valltorta (Tírig, Castellón).

co giro. Desde la ganadería extensiva tradicional, que ha dejado importantes huellas en el paisaje (azagadores, corrales, apriscos) y amplias zonas adehesadas se ha pasado a la proliferación de granjas industriales. Esta actividad está ocasionando importantes problemas ambientales derivados del poder contaminante de los residuos, sin olvidar el impacto visual de estas construcciones. La declaración del Parque deberá conllevar el establecimiento de medidas correctoras que reduzcan estos efectos.

\subsection{La gestión del Parque Cultural}

El Museu de la Valltorta (Lám. III), dependiente de la Dirección General de Promoción Cultural y PatrimonioArtístico, es el centro responsable de la gestión del Parque. El proyecto se basa en el desarrollo de tres líneas complementarias: protección, investigación y difusión.

A) Protección. Como ya hemos expuesto con anterioridad el Parque Cultural es uno de los Bienes de Interés Cultural incluidos en la Ley de Patrimonio Cultural Valenciano, y como tal su creación supone el establecimiento de unas medidas de protección. Actualmente se está concluyendo la redacción del expediente técnico que permitirá promover la incoación de declaración. Esta deberá hacerse mediante decreto del Consell a petición de la Conselleria de Cultura y Educación.

Al igual que en otros BIC, la incoación del expediente determinará la aplicación inmediata del régimen de protección previsto por la Ley para los Bienes declarados. Tras su declaración deberá redactarse un Plan Especial de Protección.

Esta protección legal, reguladora de usos en el interior del Parque, va acompañada por un servicio 


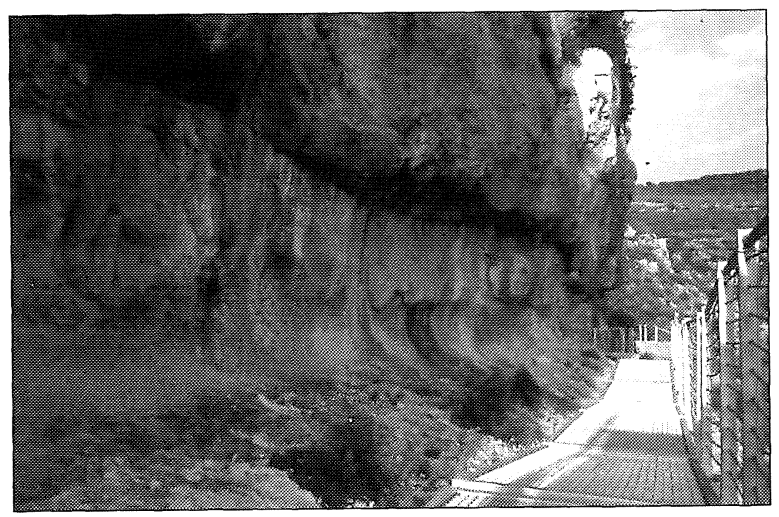

Lám. IV. Cerramiento de abrigo visitable: pasarela de acceso al conjunto del Mas d'en Josep. Barranc de la Valltorta.

de vigilancia y por la protección física del patrimonio más vulnerable: los conjuntos de arte rupestre. Pese a que contamos con un servicio de vigilantes que vela por la seguridad de los conjuntos rupestres, ha sido necesario proteger mediante vallados algunos de los abrigos. El sistema de protección física de los conjuntos del Parque está condicionado por el alto número de lugares inventariados y su diversidad en cuanto a condiciones de visibilidad, nivel de conocimiento por parte de las gentes que habitan en su entorno y accesibilidad, es decir, su consideración como lugares abiertos o no al público.

De todos los conjuntos inventariados se han seleccionado seis como lugares abiertos al público mediante visitas guiada en un horario establecido: la Cova dels Cavalls, les Coves de Ribasals o del Civil, los abrigos de La Saltadora y el Cingle del Mas d'En Josep y la Cova Remigia y el Cingle de la Mola Remigia.

En el diseño de estos cerramientos se ha intentado producir el menor impacto posible en el paisaje. Para ello se ha optado por cierres periféricos aprovechando la orografía del entorno, de manera que los propios cantiles sirvan como protección de los sitios y sólo se han instalado vallas en los puntos más vulnerables. Para facilitar el acceso hasta los abrigos visitables se han instalado pasarelas metálicas que discurren a escasa distancia de los paneles pintados. Las pasarelas cumplen una doble función; facilitan el acceso a los abrigos a un público muy heterogéneo, respecto a su facilidad para moverse por terrenos quebrados, y mantienen al público a una distancia prudente de las pinturas mientras el personal del Museo guía la visita (Lám. IV).

Los abrigos no incluidos en itinerarios de visitas y que requerían protección física se han cerra- do mediante obras de escaso impacto. En estos casos se ha evitado la apertura de nuevas rutas de acceso, ni tan siquiera bajo la justificación de tener que facilitar los trabajos de cerramiento.

B) Conservación del arte rupestre, intervenciones de conservación preventiva. Actualmente existen 33 conjuntos de arte rupestre inventariados en el Parque Cultural. La inmensa mayoría se localizan en abrigos rocosos poco profundos expuestos a la intemperie lo que ocasiona serios problemas de conservación (alteración de los soportes, instalación de colonias de hongos, líquenes y plantas vasculares, erosión eólica, incidencia de agua, efecto de incendios forestales, etc...).

A estas afecciones hay que sumar las ocasionadas por el hombre. Desde el descubrimiento de los primeros conjuntos el año 1917 hasta la actualidad buen número de estos conjuntos han sufrido agresiones antrópicas en muchos casos irreversibles como.el arrancado de figuras y graffitis por incisiones, y otras de cierta reversibilidad como pintadas, perfilado de motivos con lapiceros y mojado reiterado de las pinturas. Actualmente estas agresiones antrópicas no se producen pero todavía son patentes sus huellas en numerosos conjuntos.

Como era de esperar los conjuntos más afectados por causas antrópicas son precisamente los que fueron más frecuentados por la vistosidad de sus escenas o su accesibilidad (Cavalls, Saltadora y Ribasals). Se trata de conjuntos que generaron una dinámica desordenada de visitas, que fueron estudiados por numerosos investigadores con métodos en ocasiones muy lesivos (perfilado de figuras) y que a pesar de haber sido protegidos de forma inmediata mediante su declaración como Monumentos Histórico Artístico no gozaron de una protección real. Tal vez no hayan sido agresiones tan brutales como los arrancados de figuras de principios de siglo, pero el mojado reiterado de los paneles pintados y la acumulación de graffitis, lenta e inexorablemente terminaron por hacer ilegibles numerosos conjuntos.

El año 1987 tuvo lugar en la Valltorta una reunión de trabajo auspiciada por la Generalitat Valenciana y la Fundación Gety, sobre la conservación del arte rupestre. El encuentro contó con la presencia de prehistoriadores y restauradores. Se debatió sobre los problemas de conservación del arte rupestre al aire libre tomando como ejemplo los sufridos conjuntos de la Valltorta y se impusieron criterios conservadores respecto a las posibilidades de intervenir sobre los conjuntos rupestres. 
Diez años después, en el marco del Primer seminario internacional sobre conservación y difusión del arte rupestre al aire libre en ambiente mediterráneo que tuvo lugar en el Museu de la Valltorta, volvió a debatirse la misma cuestión. El debate surgido de este foro inspiró el inicio de un proyecto de intervenciones en los conjuntos más alterados de la Comunidad Valenciana. La primera tuvo lugar en el abrigo del Castell de Vilafamés (Vilafamés Castellón). A ella siguieron las intervenciones en la Cova dels Cavalls y en Les Coves de Ribasals o del Civil, ambos en el Barranc de la Valltorta (Lám.V).

El proyecto comenzó con el desarrollo de diversas analíticas sobre los soportes, que permitieron concretar los niveles de intervención. Los trabajos fueron desarrollados por el equipo formado por los restauradores Eudal Guillamet, Javier Chillida, Laura Ballester y Margarita Domenech.

Los trabajos se han desarrollado en dos direcciones: suprimir las patologías de origen antrópico y controlar la incidencia de los agentes naturales.

En el primer apartado se han eliminado las pintadas, así como el polvo y las sales formadas sobre los paneles pintados. En los lugares donde fueron arrancados motivos se han coloreado las lagunas para minimizar su impacto visual.

Respecto a la incidencia de los agentes naturales se han eliminado las plantas vasculares, se han fijado mediante morteros los soportes mas inestables, se han desviado las vías de agua que pudieran afectar a los paneles pintados y en el caso de Ribasals se han eliminado algunos tramos de coladas de carbonato cálcico que cubrían parcialmente algunos motivos.

C) Investigación. Uno de los aspectos más deficitarios y que ha influido de forma más negativa en el desarrollo del proyecto del Parque ha sido la escasez de estudios sobre su patrimonio, y muy especialmente sobre el arqueológico. La mejor prueba de ello es que de numerosos conjuntos de arte rupestre descubiertos el año 1917 no se disponía de documentación suficiente. Muchos de ellos fueron estudiados por varios grupos de investigadores sin que llegaran a publicarse integramente los resultados ni se hiciera entrega de la documentación generada.

Las mismas carencias encontramos en cuanto al conocimiento del contexto arqueológico de las pinturas. Los conjuntos de Valltorta y Gasulla son por muchos motivos un referente obligado en los estudios de prehistoria peninsular. Pese a haber sido objeto de estudios pioneros a los que ya nos hemos referido, actualmente la información disponible

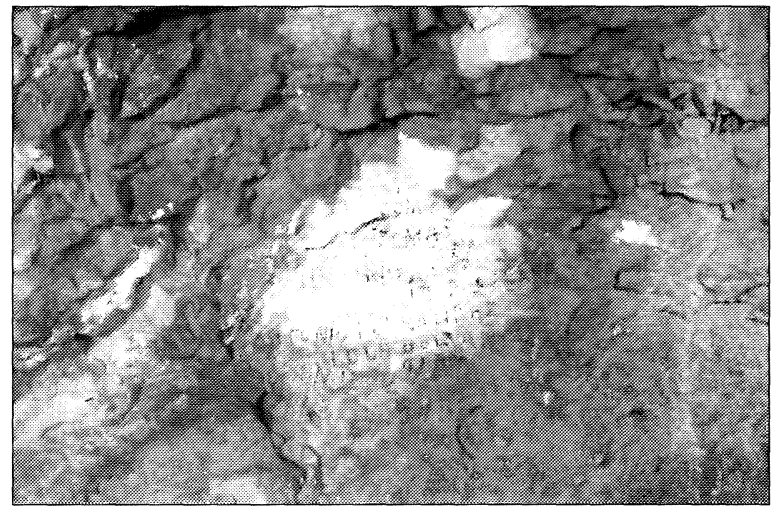

A

B

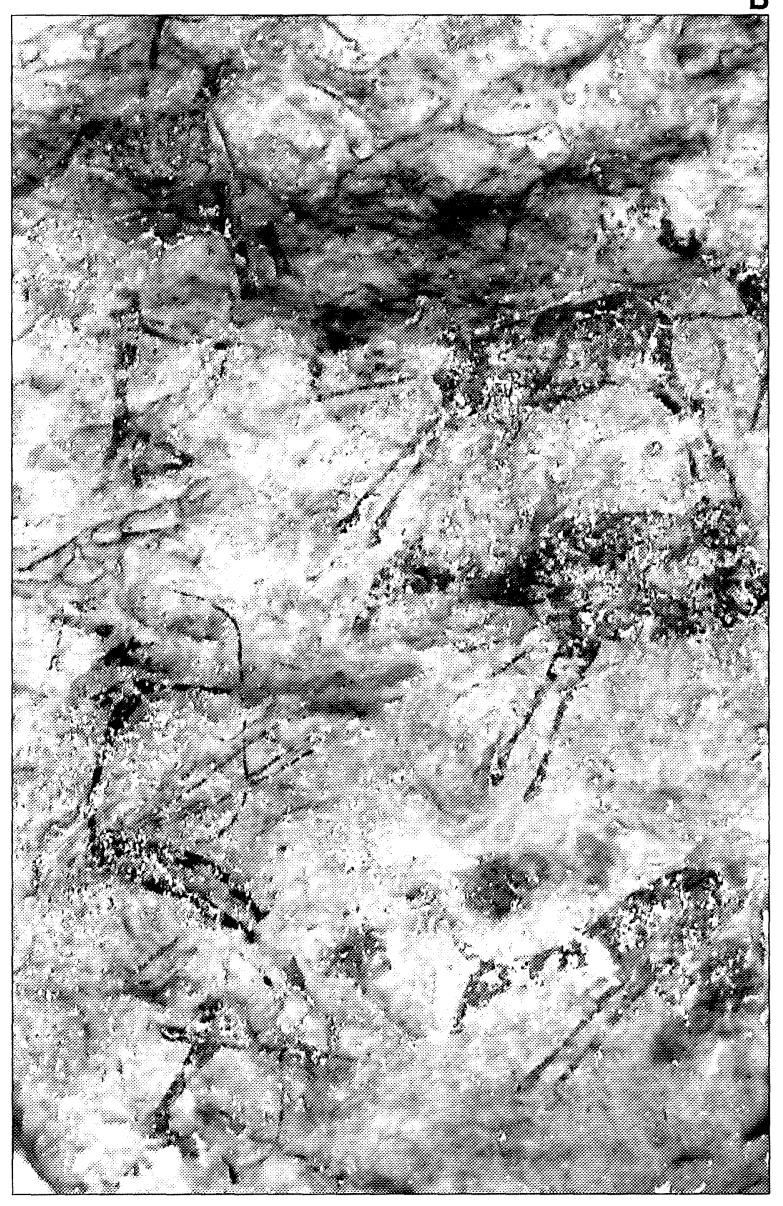

Lám. V A) Cova dels Cavalls. Estado de las pinturas antes de la intervención de conservación preventiva. B) Detalle de la escena principal después de dicha intervención.

sobre este importante conjunto arqueológico es muy parcial, como consecuencia de proceder de intervenciones arqueológicas antiguas, llevada a cabo con metodología poco rigurosa, o por estar basadas en un único yacimiento. 


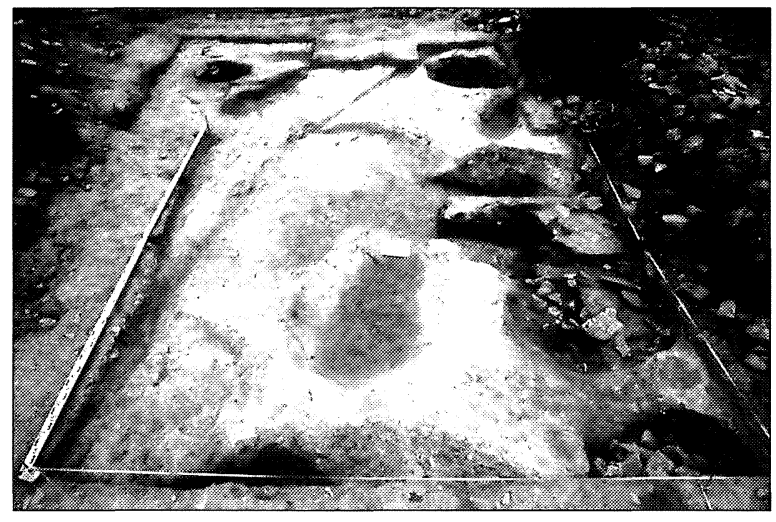

Lám. VI. Excavaciones arqueológicas en el yacimiento neolítico del Mas de Sans, Albocàsser.

Con el fin de atajar estos problemas se ha puesto en marcha el proyecto de estudio arqueológico del Parque Cultural de Valltorta-Gasulla. Se trata de un proyecto interdisciplinar coordinado por el Instituto de Arte Rupestre, con sede en el Museu de la Valltorta, que cuenta con la colaboración de investigadores de Departamentos de Prehistoria de las Universidades de Valencia y Alicante.

Los trabajos han comenzado con la verificación sobre el terreno de la información arqueológica disponible y la prospección de diversos sectores de ambas cuencas para los que se carecía de información. Estos trabajos han proporcionado el hallazgo de nuevos conjuntos de manifestaciones rupestres (pinturas y grabados) en proceso de estudio y numerosos yacimientos arqueológicos, en los que estamos llevando a cabo recogidas sistemáticas de material superficial y excavaciones (Lám.VI).

De forma paralela hemos comenzado el estudio de los conjuntos de arte rupestre con la finalidad de optimizar la documentación existente y preservar este frágil patrimonio. Esta documentación está pasando a formar parte del Centro de Documentación del Instituto de Arte Rupestre ubicado en el propio Museu de la Valltorta. En él se integra una copia del Archivo fotográfico Gil-Carles (2), adquirida por la Generalitat Valenciana el año 1992, que incluye documentación fotográfica de todos los conjuntos conocidos en el levante peninsular hasta el año 1976 y toda la documentación (calcos, fotografías, descripciones) que ha generado el estudio de los conjuntos de la Comunidad Valenciana desde entonces hasta la actualidad. Este material se

(2) El original es propiedad del CSIC y se encuentra depositado en el Dpto. de Prehistoria (Instituto de Historia, Madrid). completa con una biblioteca especializada en proceso de formación.

D) Difusión. El Museo de la Valltorta funciona como centro de acogida del Parque. El Museo, diseñado por los arquitectos Miguel del Rey e Iñigo Magro, fue inaugurado el año 1994. Cuenta con un espacio expositivo de $800 \mathrm{~m}^{2}$, distribuidos en salas de exposición permanente (Lám. VII A) y una sala de exposiciones temporales, además de una sala de proyección de audiovisuales, y diferentes áreas de trabajo entre las que se encuentra un laboratorio de arqueología, biblioteca y almacenes para materiales arqueológicos.

El Museo es el punto de partida de las visitas guiadas a lo largo de los itinerarios del Parque. Actualmente nuestra oferta consiste en la visita guiada a seis conjuntos: la Cova dels Cavalls, les Coves del Civil, la Saltadora, Mas d'En Josep , Cova Remigia y el Cingle de la Mola Remigia (Lám.VII B).

Además de estos itinerarios guiados se está diversificando la oferta con el diseño de rutas de libre acceso, con el objetivo de reducir la presión sobre los conjuntos de arte rupestre y posibilitar una aproximación a la evolución del paisaje a través de unos hitos seleccionados. Actualmente estamos trabajando en los siguientes itinerarios:

- El Cormulló dels Moros. Yacimiento Ibero-romano localizado en el nacimiento de la Valltorta. Las excavaciones desarrolladas en los últimos tres años han puesto de manifiesto la existencia de un asentamiento de los siglos II-I a.C. (Espí et alii, 2000). El proyecto prevé la musealización del yacimiento.

- La ruta de las Ermitas. Incluye una serie de construcciones religiosas dispersas, en su mayoría de los siglos XVII-XIX.

- La ruta de la arquitectura en piedra seca, itinerario en el que se incluye una muestra de las construcciones tradicionales más relevantes: casetas de volta, norias, azagadores y hornos de cal.

- La ruta de las neveras. Enlaza las tres neveras existentes en el Parque: la Nevera de la Font de Regatxols y las de la Serra y la Vella en Catí, todas ellas del siglo XVII.

- La ruta del bosque. Discurre por el interior del Barranc dels Horts, donde se conserva un robledal maduro de Quercus faginea.

- La ruta de los molinos. En el Barranc dels Molins en Ares se conservan seis molinos harineros, uno de ellos, el Moli de la Costa, todavía en uso.

Para completar la oferta, descentralizar los recur- 

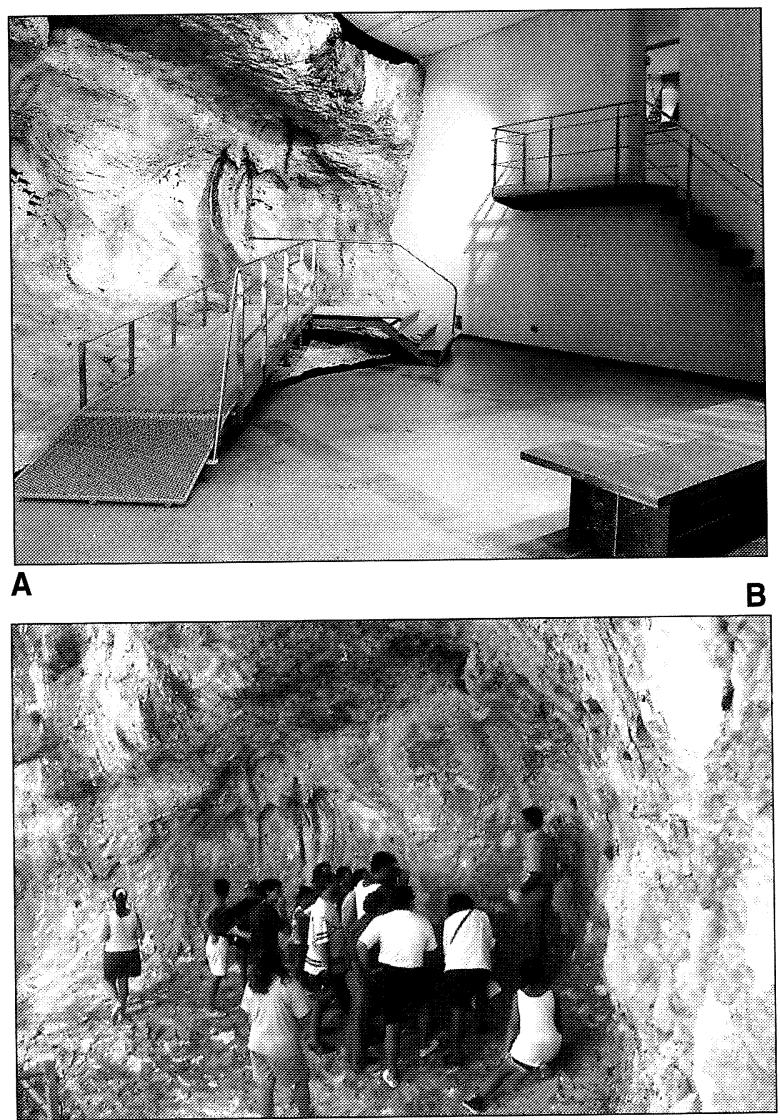

Lám. VIIA) Sala 3 del Museu de la Valltorta: réplica de la Cova dels Cavalls. B) Visita guiada a un conjunto rupestre del Barranc de la Valltorta.

sos e implicar a las poblaciones integradas en el Parque se ha previsto la creación de nuevas infraestructuras en el interior de las poblaciones: un museo de etnología en Albocàsser, un museo sobre la ganadería en Catí y otro histórico en Ares del Maestrat.

\subsection{Valoración final}

Desde su inauguración hasta la actualidad el Museo ha mantenido una media anual de 23000 visitantes. La puesta en marcha del proyecto ha supuesto:

- Un mejor conocimiento del patrimonio arqueológico del territorio, potenciado por los trabajos de investigación iniciados desde el Museo.

- Una vigilancia activa permanente sobre los conjuntos de arte rupestre.

- Un aumento de la sensibilidad social por este patrimonio.
- Una revitalización económica de la comarca desde la perspectiva del desarrollo sostenible.

La viabilidad del modelo ha llevado a la Generalitat Valenciana a promover experiencias similares en otros ámbitos de nuestra Comunidad como en Bicorp (Valencia), en los conjuntos de las Cuevas de la Araña y el Barranco Moreno.

\section{BIBLIOGRAFÍA}

AA.VV. (1990). Jornadas sobre Parques con Arte Rupestre. Dpto. de Cultura y Educación. Zaragoza.

Almagro Basch, M. (1944): "Los problemas del Epipaleolítico y Mesolítico en España”. Ampurias, VI: 1-38.

Aparicio Pérez y San VAlero (1977). La Cova Fosca (Ares del Maestre, Castellón) y el Neolítico Valenciano. Serie Arqueológica. Universidad de Valencia. Valencia.

Beltrán MARTínez, A. (1965): "Nota sobre el grupo de tres figuras negras del abrigo de La Saltadora, en el barranco de la Válltorta (Castellón)". Revista de Faculdade de Lisboa, III: 89-93.

Cabré Aguiló, J. (1923): "Las pinturas rupestres de La Valltorta I. Desaparición de las pinturas de una de las estaciones de este valle". Actas y Memorias de la Sociedad Española de Antropología, Etnografía y Prehistoria, II: 107-118.

- (1925): "Las pinturas rupestres de La Valltorta. Escena bélica de la Cova del Cevil". Actas y Memorias de la Sociedad Española deAntropología, Etnografía y Prehistoria, IV: 201-233.

CAstillo RuIz, J. (1997): El entorno de los Bienes Inmuebles de Interés Cultural. Monográfica Arte y Arqueología. Universidad de Granada. Granada.

Durán i SAMPere, A. y Pallarés, M. (1915-20): "Exploración arqueológica al Barranc de la Valltorta". Anuari de L'Institut d'Estudis Catalans, VI: 451-454.

Espí, I.; Iborra, M. ${ }^{a}$ P. y Haro, S. de (2000): "El área de almacenaje del poblado ibero-romano del Cormulló dels Moros, Albocàsser, Castelló". Saguntum, extra III: 149-152.

González Prats, A. (1979): Carta arqueológica del Alto Maestrazgo. Trabajos Varios del S.I.P., 63. Valencia.

Gusi Jener, F. (1975a): "Las dataciones de C14 de la Cueva del Mas d'Abad (Coves de Vinromà). Campaña arqueológica 1975. Ensayo cronológico para la periodización del Bronce Valenciano". Cuadernos de Prehistoria y Arqueología Castellonense, 2: 75-79.

- (1975b): "Un taller de sílex bajo abrigo en la segunda cavidad del Cingle de la Ermita(Albocàsser)". Cuadernos de Prehistoria y Arqueología Castellonense, 2: 39-63.

Gusi Jener, F. y Olaria de Gusi, C. (1975): "La cerámica de la Edad del Bronce de la Cueva del Mas d'Abad (Coves deVinrromà). Campaña arqueológica 1975. Cam- 
pañaArqueológica, 1975". Cuadernos de Prehistoria y Arqueología Castellonense, 2: 103-115.

HERNÁNDEZ PACHECO, E. (1918): Estudios de arte prehistórico. Prospecciones de las pinturas de Morella la Vella. Evolución de las ideas madres de las pinturas rupestres. Comisión de Investigaciones Paleontológicas y Prehistóricas, Memoria,16. Madrid: 1-25.

Maluquer de Motes, J. (1939): "Las industrias con microburiles de la Valltorta". Ampuries, I: 108-112.

Mesado Oliver, N. (1981): "La Cova del Mas d'En Llorenç y el arte prehistórico del barranco de Gasulla". Archivo de Prehistoria Levantina, XVI: 281-306.

OBERMAIER, H. y WERNERT, P. (1919): Las pinturas rupestres del Barranco de la Valltorta. Comisión de Investigaciones Paleontológicas y Prehistóricas. Memoria, 23. Madrid.

Olaria, C. (1988): Cova Fosca. Un asentamiento mesoneolítico de cazadores y pastores en la serranía delAlto Maestrazgo. Monografíes del Prehistòria i Arqueologia Castellonenques

Pallarés, M. (1915-20): "Exploracio dels jaciments prehistorics de la Valltorta”. Anuari de L'Institut d'Estudis Catalans, VI: 454-457.

Porcar, J.B. (1934): "Pintures Rupestres al Barranc de Gasulla". Boletín de la Sociedad Castellonense de Cultura, XV, VI: 343-347.

- (1935): "Noves pintures rupestres en el terme d'Ares". Boletín de la Sociedad Castellonense de Cultura, XVI, I: 30-32.

- (1945): "Iconografía rupestre de la Gasulla y Valltorta. (Danza de arqueros ante figuras humanas sacrificadas)". Boletín de la Sociedad Castellonense de Cultura, XXI: 145-178. Castellón.

- (1946): "Iconografía rupestre de la Gasulla y Valltorta. Escenas bélicas". Boletín de la Sociedad Castellonense de Cultura, XXII: 48-60.

- (1947): "Iconografía rupestre de Gasulla y Valltorta. Representación pictográfica del toro. Sus características. Particularidades que ofrece". Boletín de la Sociedad Castellonense de Cultura, XXIII: 314-324.

- (1949a): "Iconografía rupestre de Gasulla. Representaciones de insectos. Características y particularidades que ofrece". Boletín de la Sociedad Castellonense de Cultura, XXV: 169-182.

- (1949b): "Interpretaciones y sugerencias en torno a las pinturas rupestres del abrigo décimo del Cingle de Mola Remigia". Boletín de la Sociedad Castellonese de Cultura, XXV: 642-648.

- (1949c): "Noves pintures rupestres en el terme d'Ares del Maestre". Boletín de la Sociedad Castellonese de Cultura, XXV: 144.

Porcar, J.B.; Obermaier, H. y Breull, H. (1936): Las pinturas rupestres de la Cova Remigia (Castellón). Junta Superior del Texto Artístico. Madrid.

Querol, M.A. (1993): "Filosofía y concepto de Parque Arqueológico". Seminario de Parques Arqueológicos. Ministerio de Cultura. Madrid: 13-22.

Rivière, G.H. (1993): La Museología. Curso de museología. Textos y testimonios. Akal.Arte y Estética. Madrid.

Ripoll Perelló, E. (1963): Pinturas Rupestres de La Gasulla (Castellón). Instituto de Prehistoria y Arqueología de la Diputación Provincial de Barcelona yWenner Gren Foúndation forAnthropological Research. Barcelona.

- (1970): "Noticia sobre l'estudi de les pintures rupestres de La Saltadora". Cuadernos deArqueología e Historia de la Ciudad, XIV: 9-24.

VAL, M. J. de (1977): "Yacimientos líticos de superficie en el Barranco de la Valltorta (Castellón)". Cuadernos de Prehistoria y Arqueología Castellonense, 4: 45-77.

VIÑAs, R. (1970): "Peligro en las pinturas delArte levantino, Barranco de la Valltorta (Castellón)". Speleon, 18: 75-79.

- (1978): "Informe sobre un microorganismo detectado en las pinturas rupestres del Barranco de la Valltorta". Cuadernos de Prehistoria y Arqueología Castellonense, 5: 361-367.

- (1979-90): "Figuras inéditas del Barranco de La Valltorta". Ampurias, 41-42: 1-34.

- (1981): "Noticia de un nuevo conjunto de pinturas rupestres enAlbocàsser". Cuadernos de Prehistoria yArqueología Castellonense: 301-305.

- (1982): La Valltorta. Ediciones Castell. Barcelona.

T. P., 57, n. ${ }^{\circ} 2,2000$ 